\title{
Nível de conhecimento tático declarativo, a posição de jogo e o tempo de reação em atletas de handebol
}

\author{
Level of declarative tactical knowledge, the game position \\ and the time of reaction in handball athletes
}

\author{
Nivel de conocimientos tácticos declarativos, la posición del \\ juego y el tiempo de reacción en os atletas de bolsillo
}

\author{
Iberê Caldas Souza Leão ${ }^{1}$, Luvanor Santana da Silva ${ }^{1}$, Eduarda \\ Francini Alves Barbosa ${ }^{1}$, Edil de Albuquerque Rodigues Filho ${ }^{1,2}$
}

1.Núcleo de Educação Física, Centro Acadêmico de Vitória, Universidade Federal de Pernambuco, VitóriaPE, Brasil.

2.Faculdade do Recife, PE, Brasil.

\begin{abstract}
Resumo
Introdução. O conhecimento tático declarativo (CTD) faz parte das habilidades cognitivas presentes nos esportes coletivos. Objetivo. Avaliar o nível de CTD de 60 atletas de handebol e comparar respostas quanto à posição de jogo e o tempo de reação. Método. Estudo descritivo, transversal e comparativo, com amostras por conveniência. Utilizou-se a média, desvio padrão e o nível de significância $p<0,05$. Resultados. Os indivíduos apresentaram um "potencial em evolução" para o nível de CTD e quando comparado com a posição de jogo não apresentou resultado significativo $(p=0,82)$. O CTD e o tempo de reação, encontrou-se diferença significativa apenas para as atletas de Pernambuco $(p \leq 0,01)$; o tempo de reação em comparação com a posição de jogo, encontrou-se diferença significativa entre as seguintes posições: Goleira $x$ ponta $(p=0,03)$; ponta $x$ meia $(p=0,02)$; ponta $x$ pivô $(p \leq 0,01)$ e central $x$ pivô $(p=0,04)$. Sendo as centrais e as goleiras as atletas que apresentaram os melhores tempos de reação. Conclusão. Concluímos que as atletas se encontram com nível de CTD em evolução, não tendo influência da posição de jogo; as atletas de Pernambuco foram mais rápidas em suas decisões, e ainda, as centrais, goleiras e pontas apresentaram melhores tempos de reação do que as meias e pivôs.
\end{abstract}

Unitermos. Esporte; Cognição; Tomada de decisão; Desempenho atlético

\begin{abstract}
Introduction. Declarative tactical knowledge (DTK) is part of the cognitive abilities present in collective sports. Objective. It was to evaluate the DTK level of 60 handball players and to compare responses regarding game position and reaction time. Method. Descriptive, cross-sectional and comparative study with samples for convenience. The mean, standard deviation and significance level were set at $p<0.05$. Results. The individuals presented an "evolving potential" for the DTK level, and when compared with the playing position, did not present significant result $(p=0.82)$. The DTK and the reaction time, found a significant difference only for the athletes of Pernambuco $(p \leq 0.01)$; the reaction time in comparison with the game position, we found a significant difference between the following positions: Goalie $x$ tip $(p=0.03)$; tip $x$ half $(p=0.02)$; tip $\times$ pivot $(p \leq 0.01)$, and center $\times$ pivot $(p=0.04)$. Being the central and the goalkeepers were the athletes who presented the best times of reaction. Conclusion. We conclude that the athletes are with a level of DTK in evolution, having no influence of the game position; the athletes of Pernambuco were faster in their decisions, and still, the central, goal and goal had better times of reaction than the socks and pivots.

Keywords. Sport; Cognition; Decision-making; Tactics; Athletic performance
\end{abstract}




\section{Resumen}

Introdución. El conocimiento táctico declarativo (CTD) es parte de las habilidades cognitivas presentes en los deportes colectivos. Objetivo. El objetivo era evaluar el nivel de CTD de 60 jugadores de balonmano y comparar las respuestas con respecto a la posición del juego y el tiempo de reacción. Método. Estudio descriptivo, transversal y comparativo con muestras por conveniencia. La media, la desviación estándar y el nivel de significación se establecieron en $\mathrm{p}<0,05$. Resultados. Los individuos presentaron un "potencial evolutivo" para el nivel de CTD y, en comparación con la posición de juego, no presentaron un resultado significativo $(p=0,82)$. El CTD y el tiempo de reacción encontraron una diferencia significativa solo para los atletas de Pernambuco $(p \leq 0,01)$; el tiempo de reacción en comparación con la posición del juego, encontramos una diferencia significativa entre las siguientes posiciones: Goalie $x$ tip $(p=0,03)$; punta $x$ media $(p=0,02)$; punta $x$ pivote $(p \leq 0.01)$ y centro $x$ pivote $(p=0,04)$. Siendo el centro y los porteros fueron los atletas que presentaron los mejores momentos de reacción. Conclusión. Concluimos que los atletas están con un nivel de CTD en evolución, sin tener influencia de la posición del juego; los atletas de Pernambuco fueron más rápidos en sus decisiones, y aún así, la meta central y la meta tuvieron mejores tiempos de reacción que los calcetines y los pivotes.

Palabras clave. Deporte; Cognición; Toma de decisiones; Rendimiento atlético

Trabalho realizado na Universidade Federal de Pernambuco, Vitória-PE, Brasil.

\section{INTRODUÇÃO}

Dentro do parâmetro esporte e competição, dentre os jogos esportivos coletivos (JEC) podemos destacar 0 handebol, sendo uma modalidade caracterizada por apresentar um ambiente de grande imprevisibilidade, dinâmico, necessitando dos atletas rápidas tomadas de decisões, nos momentos de oposição entre as equipes que atuam com os mesmos objetivos: Em que um deles é marcar mais gols que a equipe adversária ${ }^{1}$.

Para o desenvolvimento do handebol, se faz necessário um adequado processo de ensino-aprendizagemtreinamento (E-A-T), no qual diz respeito ao entendimento dos processos cognitivos por parte dos treinadores e atletas $^{2}$. Conceitualmente esses processos são definidos 
como estruturas mentais que tem estreita relação com a consciência e o conhecimento, isto é, a percepção, o pensamento, a antecipação, a atenção e a tomada de decisão ${ }^{1}$.

A capacidade de tomar decisão depende de alguns fatores como orientação, que pode vir do treinador/professor, sintonia com o meio ambiente e as próprias habilidades do indivíduo ${ }^{3}$. As ações do jogo podem ser condicionadas por pressões e interações externas (posicionamentos e movimentações dos colegas, espaço de jogo, comportamento defensivo, relação com objetivo e alvo, trajetória dos jogadores, etc.) e internas (nível de motivação e esforço $)^{4}$. Embora para que possa atingir alto nível de aproveitamento, os estímulos dos treinos deverão ser similares as demandas situacionais do próprio jogo ${ }^{5}$.

Durante a prática de uma modalidade esportiva coletiva (MEC), vários processos cognitivos são despertados no seu desenrolar, fazendo necessário que os seus participantes tomem atitudes táticas e estratégicas, usando seu conhecimento ${ }^{6}$. Sendo assim, os processos cognitivos são de extrema importância para obtenção de um bom desempenho por parte dos atletas ${ }^{6}$.

A execução de uma técnica desportiva em situação de jogo pode ser resultado do conhecimento tático adquirido ${ }^{4}$. O conhecimento tático é de extrema importância para que o atleta possa compreender o jogo, e assim tomar a decisão mais adequada no momento certo, considerando que esse conhecimento não é geral e sim específico da modalidade, 
ou seja, o conhecimento tático é inerente a modalidade praticada ${ }^{7}$.

Diversos autores enfatizam que nas ciências dos esportes são encontrados dois tipos de conhecimento tático, o conhecimento tático declarativo (CTD) e o conhecimento tático processual $(\mathrm{CTP})^{7-10}$. O CTD está relacionado com a capacidade do atleta saber "o que fazer", ou seja, conseguir declarar verbalmente ou através da escrita qual a melhor decisão a ser tomada e o porquê dessa decisão. Enquanto o CTP refere-se ao "como fazer", ou seja, a operacionalização da ação, dessa forma estando mais ligado ao gesto motor, em que esses dois conhecimentos interagem naturalmente ${ }^{11,12}$. A literatura tem demonstrado que o CTD está relacionado com algumas variáveis como idade, nível de experiência, nível competitivo dos campeonatos, etc ${ }^{13-15}$.

Diante do que foi descrito, o objetivo desse estudo foi avaliar o nível de conhecimento tático declarativo de jogadores de handebol de três estados do nordeste (Pernambuco-PE, Alagoas-AL, Rio Grande do Norte-RN), além de comparar suas respostas quanto à posição de jogo e o tempo de reação.

\section{MÉTODO}

\section{Amostra}

O estudo foi do tipo descritivo, transversal e comparativo, com amostras escolhidas por conveniência; 
foram selecionados 60 atletas da categoria adulta feminino de três estados da região Nordeste do Brasil ( $P E, A L, R N)$.

Este estudo respeitou as normas estabelecidas pelo Conselho Nacional em Saúde (466 - 2012) envolvendo pesquisa com seres humanos, a partir da aprovação do comitê de ética da Universidade Federal de Pernambuco sob o protocolo CAEE: 55174116600005208.

\section{Procedimento}

A coleta ocorreu sempre momentos antes da sessão do treinamento, no local em que os atletas treinaram, em Novembro de 2014. Foi utilizado o protocolo validado para verifica níveis de percepção e tomada de decisão (CTD dos atletas) ${ }^{7}$.

O protocolo utilizado é constituído de 11 cenas de vídeo do jogo de handebol (situações ofensivas do jogador atacante com bola). O indivíduo realizou o teste em um equipamento eletrônico (tablet) em que o mesmo analisou as 11 cenas citadas anteriormente.

Cada cena durou 7 a 10 segundos e, ao final de cada uma, houve um congelamento por três segundos e, durante este tempo, o indivíduo testado teve que raciocinar e tomar uma decisão do que ele faria (situação problema do jogo). As opções (decisões) de escolha eram: Passar ( ); Fintar ( ) e Arremessar ( ).

Ao final, foi contabilizado o número de acertos (nível de CTD), como também o potencial e tempo de reação do indivíduo no teste (Tabela 01). 
Tabela 1. Potenciais relacionados ao número de acertos no teste (nível de CTD no handebol).

\begin{tabular}{ccccc}
\hline & \multicolumn{4}{c}{ POTENCIAIS } \\
\hline Potencial Fraco & Potencial em & Potencial & Potencial & Potencial \\
& evolução & Médio & Acima da média & excelente \\
\hline $0-2$ & $3-5$ & $6-7$ & $8-9$ & 10 \\
Acertos & Acertos & Acertos & Acertos & Acertos \\
\hline
\end{tabular}

A partir do número de acertos obtidos pelo indivíduo atleta no teste e por seu enquadramento em um dos potenciais acima citados, também foram utilizadas frases para que o atleta se sentisse estimulado a evoluir no treino e na competição (Tabela 2).

Tabela 2. Potenciais obtidos e frases estimuladoras para os atletas.

\begin{tabular}{cl}
\hline POTENCIAIS & \multicolumn{1}{c}{ FRASES ESTIMULADORAS } \\
\hline $0-2$ Potencial fraco & $\begin{array}{l}\text { Não está conseguindo interpretar as } \\
\text { situações problemas do jogo; procure treinar } \\
\text { mais atento e focado nas situações ofensivas. }\end{array}$ \\
\hline $3-5$ Potencial em evolução & $\begin{array}{l}\text { Melhore suas decisões de acordo com as } \\
\text { situações problemas que surgem durante o } \\
\text { jogo. }\end{array}$ \\
\hline $6-7$ Potencial médio & $\begin{array}{l}\text { Decidi adequadamente em algumas situações } \\
\text { problemas do jogo; melhore sua percepção e } \\
\text { tenha mais atenção no treino das ações } \\
\text { táticas ofensivas. }\end{array}$ \\
\hline $8-9$ Potencial acima da média & $\begin{array}{l}\text { Percebe e toma decisões adequadas nas } \\
\text { situações do jogo, não se acomode continue } \\
\text { evoluindo. }\end{array}$ \\
\hline 10 Potencial excelente & $\begin{array}{l}\text { Percebe e toma decisões eficazes; mantenha } \\
\text { seu potencial. }\end{array}$ \\
\hline
\end{tabular}

\section{Analise estatística}

Para as análises descritivas, foram utilizadas as medidas de média e desvio padrão e a distribuição de probabilidades (análise percentual). Os dados foram submetidos ao tratamento de distribuição normal 
(Komogorov-Smirnov) e homogeneidade de variância (Bartlet), utilizando-se ainda a correlação de Pearson, correlação de Spearman, teste de Kruskal-wallis e posteriormente o Post Hoc de Student Newman Keuls, com o nível de significância $p<0,05$. Os dados foram gerados pelo pacote estatístico SPSS for Windows - versão 17.0; 2007.

\section{RESULTADOS}

Os resultados abaixo dizem respeito à análise do nível de CTD das amostras avaliadas, comparando com a posição de jogo e o tempo de reação das atletas (Tabelas 3, 4 e 5).

Tabela 3. Perfil do nível de CTD das atletas de handebol, categoria adulta feminina de três estados do Nordeste do Brasil.

\begin{tabular}{|c|c|c|c|c|}
\hline \multicolumn{5}{|c|}{ VARIÁVEIS } \\
\hline Posição & Amostras & $\begin{array}{c}\text { Distribuição de } \\
\text { probabilidades }(\%)\end{array}$ & $\begin{array}{c}\text { Nível de CTD } \\
\text { (no de acertos) }\end{array}$ & Potenciais \\
\hline Goleiro & 7 & 11,67 & $\begin{array}{c}2 \\
33445 \\
7 \\
\end{array}$ & $\begin{array}{c}\text { Fraco } \\
\text { Em evolução } \\
\text { Médio }\end{array}$ \\
\hline Central & 7 & 11,67 & $\begin{array}{c}2 \\
33345 \\
6\end{array}$ & $\begin{array}{c}\text { Fraco } \\
\text { Em evolução } \\
\text { Médio }\end{array}$ \\
\hline $\begin{array}{c}\text { Meias } \\
\text { (Direita/Esquerda) }\end{array}$ & 16 & 26,67 & $\begin{array}{c}11222 \\
33334455 \\
666\end{array}$ & $\begin{array}{c}\text { Fraco } \\
\text { Em evolução } \\
\text { Médio }\end{array}$ \\
\hline Pivô & 10 & 16,67 & $\begin{array}{c}22 \\
33334455\end{array}$ & $\begin{array}{c}\text { Fraco } \\
\text { Em evolução }\end{array}$ \\
\hline $\begin{array}{c}\text { Pontas } \\
\text { (Direita/Esquerda) }\end{array}$ & 20 & 33,33 & $\begin{array}{c}1122 \\
33333344 \\
4555555 \\
6\end{array}$ & $\begin{array}{c}\text { Fraco } \\
\text { Em evolução } \\
\text { Médio }\end{array}$ \\
\hline Total & 60 & $100 \%$ & 210 & ----- \\
\hline Média & 8,5 & ---- & ----- & Em evolução \\
\hline $\begin{array}{c}\text { Desvio Padrão } \\
p \geq 0,82\end{array}$ & 4,2 & ---- & ---- & ----- \\
\hline
\end{tabular}


Tabela 4. Comparação do nível de conhecimento tático declarativo (CTD) e o tempo de reação (TR) das atletas entrevistadas.

\begin{tabular}{lc}
\hline \multicolumn{1}{c}{ CTD $\times$ TR / Estado } & P \\
\hline \multicolumn{1}{c}{$\mathrm{N}=60$} & 0,04 \\
\hline Alagoas $-\mathrm{AL}$ & 0,99 \\
\hline Pernambuco $-\mathrm{PE}$ & 0,01 \\
\hline Rio Grande do Norte $-\mathrm{RN}$ & 0,23 \\
\hline
\end{tabular}

Tabela 5. Comparação do tempo de reação e a posição de jogo das atletas entrevistadas.

\begin{tabular}{cc}
\hline Tempo de Reação X Posição de jogo & p \\
\hline Goleira x Ponta & 0,03 \\
\hline Goleira x Meia & 0,76 \\
\hline Goleira x Central & 0,07 \\
\hline Goleira x Pivô & 0,97 \\
\hline Ponta x Meia & 0,02 \\
\hline Ponta x Central & 0,90 \\
\hline Ponta x Pivô & 0,01 \\
\hline Meia x Central & 0,06 \\
\hline Meia x Pivô & 0,70 \\
\hline Central x Pivô & 0,04 \\
\hline
\end{tabular}

\section{DISCUSSÃO}

Os nossos resultados constituem-se na essência da prática do handebol, privilegiando a variabilidade que se insere 0 indivíduo no ambiente do jogo (estímulos e decisões) ${ }^{16}$, nesse estudo o nível de CTD não teve relação com a posição de jogo em que atuavam os atletas. Em um estudo sobre futebol, não foram encontradas diferenças entre o CTD e a posição de jogo nessa modalidade, corroborando com a nossa investigação ${ }^{17}$.

Observando a Tabela 1, a maioria dos indivíduos encontra-se com potencial em evolução, esses resultados nos mostram que as atletas ainda devem evoluir no que diz respeito aos seus níveis de percepção e tomada de decisão; vale salientar, que essa foi a primeira vez que esse grupo 
de jogadoras foram testadas com um instrumento dessa natureza, em que as mesmas não tinham conhecimento sobre a existência de dois tipos de conhecimentos envolvidos com a prática do handebol, o CTD e o CTP ${ }^{18}$.

Tanto as atletas como o treinador, conhecendo o CTD e o CTP; o entendimento e a aplicação prática do processo de E-A-T do handebol favorecerá uma melhor compreensão de todos que compõem a equipe; em que o treinador terá que ser responsável por utilizar em seus treinos, métodos de ensino que enfatizem os processos cognitivos inerentes à modalidade, esses provavelmente, propiciarão junto às atletas boas tomadas de decisões nas situações problemas que surgem durante a competição $3,19,20$.

Aliado ao CTD, analisamos o tempo de reação (TR) das atletas, e após as análises agrupadas dos indivíduos $(n=60)$, percebemos que existe uma diferença em seus resultados $(p=0,04)$ no que diz respeito à comparação entre essas duas variáveis, desta maneira, identificamos também, que o resultado do grupo de jogadoras Pernambucanas influenciou nos resultados de todo o grupo investigado $(p \leq 0,01)$. O tempo ou a velocidade de reação é a capacidade que possui um indivíduo atleta em reagir a um estímulo externo em um menor espaço de tempo possível $^{21}$.

O TR que um indivíduo tem para responder a estímulo visual é entre $109-250 \mathrm{~ms}$ masculino, quanto que feminino é de $121 \mathrm{~ms}$, nesse estudo a atleta teve 3 segundos para tomar uma decisão para cada cena de vídeo apresentada ${ }^{22}$. 
Observou-se que as atletas de Pernambuco se apresentaram mais rápidas em suas decisões no teste do nível de CTD tendo melhor aproveitamento quando comparado outros estados, mas, essa resposta deve-se a execução das atividades no momento do treino? Ou aos métodos de ensino que propiciam execuções rápidas dos gestos técnicos dentro das situações problemas ${ }^{22}$ ? Uma proposta de análise do treinamento onde se podem analisar melhor vários estímulos e as atividades executadas no treino utilizados pelo treinador na busca da comprovação dessa íntima relação entre CTD x TR.

O TR além de ser influenciado pelo nível de atividade física habitual e esporte praticado, se diferencia conforme os estímulos se apresentam, por exemplo, e tempo de resposta para um estímulo visual é mais longo do que uma resposta a um estímulo auditivo, pois mudança da energia da luz para impulsos neurais que podem ser levados ao cérebro pela retina ocorre de forma mais lenta, que a transformação das ondas de energia em impulsos que estimulam o sistema auditivo ${ }^{22}$.

Quando comparamos o TR das atletas com posição de jogo, verificamos que algumas das jogadoras se apresentaram mais rápidas do que outras dentro da equipe, isso confirma o que tem sido discutido na prática do handebol, a goleira, por exemplo, tem que apresentar um alto TR para intervir frente aos potentes arremessos das várias posições de jogo; nesse estudo as goleiras, as pontas e as centrais apresentaram os melhores TR de toda 
amostra. O melhor desempenho das goleiras no TR deve-se aos programas específicos de treino para essa posição, diferentemente das atividades que são submetidas às outras atletas, sem diferenciação quanto a posição de jogo $^{23}$.

Entretanto, é importante salientar que a análise (testes) de habilidades cognitivas (percepção e tomada de decisão) e posteriormente motoras (TR), deve-se adequar ao máximo as ações técnico/táticas ao ambiente do jogo; o que foi considerado nesse estudo.

\section{CONCLUSÃO}

Dessa forma, concluímos que as atletas investigadas se encontram com nível de CTD em evolução e essa variável não sofreu influência da posição de jogo; como também, as atletas de Pernambuco apresentaram-se mais rápidas em suas decisões do que as atletas dos outros estados, e ainda, as centrais, goleiras e pontas apresentaram melhores tempos de reação do que as armadoras laterais (meias) e pivôs.

\section{REFERÊNCIAS}

1.Matias CJAS, Greco PJ. Cognição \& Ação nos jogos esportivos coletivos. Ciências \& Cognição 2010;15:252-71.

http://www.cienciasecognicao.org/pdf/v15 1/m123 09.pdf

2.Menezes RP, Marques RFR, Nunomura M. Especialização esportiva precoce e o ensino dos jogos coletivos de invasão. Movimento 2014;20:351-73. https://doi.org/10.22456/1982-8918.40200 
3. Mesquita I, Garganta J, Afonso J. A tomada de decisão no desporto: - papel da atenção, da antecipação e da memória. Rev Bras Cineantropometria Desemp Hum 2012;14:592-601. https://doi.org/10.5007/1980-0037.2012v14n5p592

4.Greco PJ, Aburachid LMC, Silva SRd, Morales JCP. Validação de conteúdo de ações tático-técnicas do Teste de Conhecimento Tático Processual: Orientação Esportiva. Motricidade 2014;10:38-48. http://dx.doi.org/10.6063/motricidade.10(1).2124

5.Silva MV, Praça GM, Torres CG, Greco PJ. Comportamento tático individual de atletas de Futebol em situações de Pequenos Jogos. $R$ Min Educ Fís Viçosa 2013;9:676-83.

https://www.academia.edu/32483251/COMPORTAMENTO T\%C3\%81 TICO INDIVIDUAL DE ATLETAS DE FUTEBOL EM SITUA\%C3\%87 $\%$ C3\%95ES DE PEQUENOS JOGOS

6. Menezes RP. Contribuições da concepção dos fenômenos complexos para o ensino dos esportes coletivos. Motriz: Rev Educ Fís 2012;18:34-41. http://dx.doi.org/10.1590/S198065742012000100004

7.Caldas I, Viana M, Greco P, Sougey E. Construção de um protocolo do nível de conhecimento tático declarativo no handebol. Rev Min Edu Fís (UFV) 2013;9:1108-14.

8.Silva NIA, Viana MT, Leão ICS. Análise do nível de conhecimento tático declarativo no handebol feminino. Rev Bras Esp Col 2018;2:5267.

https://periodicos.ufpe.br/revistas/esportecoletivo/article/view/23924 5/30926

9.Costa GDCT, Castro HdO, Cabral FdA, Morales JCP, Greco PJ. Content Validity of scenes of the Declarative Tactical Knowledge Test in Volleyball-DTKT: Vb. Rev Bras Cineantropom Desempenho Hum 2016;18:629-37.

http://dx.doi.org/10.5007/1980-0037.2016v18n6p629

10.Gil A, Moreno MP, García-González L, Moreno A, del Villar F. Analysis of declarative and procedural knowledge in volleyball according to the level of practice and players' age. Percep Mot Skills 2012;115:632-44.

http://dx.doi.org/10.2466/30.10.25.PMS.115.5.632-644

11.Memmert D. Inattentional blindness to unexpected events in 815-year-olds. Cog Develop 2014;32:103-9.

https://doi.org/10.1016/j.cogdev.2014.09.002

12. Aburachid LM, Morales JC, Greco PJ. Test validation process of tactical knowledge in tennis: the influence of practice time and competitive experience. Inter J Sports Sci 2013;3:13-22. https://doi.org/10.5923/j.sports.20130301.04

13. Aburachid LMC, da Silva SR, Greco PJ. Nível de conhecimento tático de jogadores e avaliação subjetiva dos treinadores no futebol. RBFF Rev Bras Futsal Futebol 2013;5:322-30.

http://www.rbff.com.br/index.php/rbff/article/view/218/204

14. de Oliveira Castro H, Cavalli I, da Silva Matias CJA, Mendes JC, Greco PJ. Relação entre o conhecimento tático declarativo e 
classificação final de equipes juvenis masculinas de voleibol. ACTA Bras Mov Hum 2015;5:64-79.

http://www.periodicos.ulbra.br/index.php/actabrasileira/article/view/ $\underline{2837 / 2103}$

15.Amaral SG, Greco JP, Monteiro GN, Mazzardo T, Araújo ND, Aburachid LMC. Tactical knowledge of handball players considering time of practice and position in the competition. Rev Bras Cineantropom Desempenho Hum 2018;20:309-17.

http://dx.doi.org/10.5007/1980-0037.2018v20n3p309

16.Scaglia AJ, Reverdito RS, Leonardo L, Lizana CJR. O ensino dos jogos esportivos coletivos: as competências essenciais e a lógica do jogo em meio ao processo organizacional sistêmico. Movimento 2013;19:227-49.

https://www.seer.ufrgs.br/Movimento/article/viewFile/37893/27534

17.Giacomini DS, Silva EG, Greco PJ. Declarative tactical knowledge in soccer: a comparison study between soccer players of different categories and positions. Rev Bras Ciênc Esporte 2011;33:445-63.

http://dx.doi.org/10.1590/S0101-32892011000200011

18.Giacomini DS, Soares V, Santos HF, Matias CJ, Greco PJ. O conhecimento tático declarativo e processual em jogadores de futebol de diferentes escalões. Motri 2011;7:43-53.

http://www.scielo.mec.pt/pdf/mot/v7n1/v7n1a06.pdf

19. Matias CJAdS, Greco PJ. O conhecimento tático declarativo dos levantadores campeões de voleibol. Motriz 2013;19:184-94. http://www.scielo.br/pdf/motriz/v19n1/a19v19n1.pdf

20.Pombo Menezes R, Baldy dos Reis $\mathrm{HH}$, Tourinho Filho H. Ensinoaprendizagem-treinamento dos elementos técnico-táticos defensivos individuais do handebol nas categorias infantil, cadete e juvenil. Movimento 2015;21:261-73. https://doi.org/10.22456/1982$\underline{8918.46159}$

21.Caldas I. Treinando handebol. Recife: Editora da Universidade Federal de Pernambuco. 2014.

22.Bruzi AT, Fialho JVAP, Fonseca FdS, Ugrinowitsch H. Comparison of reaction time between Basketball players, Artistic Gymnasts and non-athletes. Rev Bras Ciênc Esporte 2013;35:469-80. http://dx.doi.org/10.1590/S0101-32892013000200015

23.Ruschel C, Haupenthal A, Hubert M, Fontana H, Pereira S, Roesler $\mathrm{H}$. Tempo de reação simples de jogadores de futebol de diferentes categorias e posições. Motri 2011;7:73-82.

http://www.scielo.mec.pt/pdf/mot/v7n4/v7n4a08.pdf 